\title{
Local analysis of the feasible primal-dual interior-point method
}

\author{
R. Silva · J. Soares $\cdot$ L.N. Vicente
}

Received: 21 September 2005 / Revised: 1 August 2006 / Published online: 16 October 2007

(C) Springer Science+Business Media, LLC 2007

\begin{abstract}
In this paper we analyze the rate of local convergence of the Newton primal-dual interior-point method when the iterates are kept strictly feasible with respect to the inequality constraints.

It is shown under the classical conditions that the rate is q-quadratic when the functions associated to the binding inequality constraints are concave. In general, the q-quadratic rate is achieved provided the step in the primal variables does not become asymptotically orthogonal to any of the gradients of the binding inequality constraints.

Some preliminary numerical experience showed that the feasible method can be implemented in a relatively efficient way, requiring a reduced number of function and derivative evaluations. Moreover, the feasible method is competitive with the classical infeasible primal-dual interior-point method in terms of number of iterations and robustness.
\end{abstract}

Keywords Interior-point methods $\cdot$ Strict feasibility $\cdot$ Centrality $\cdot$ Local convergence

R. Silva is Ph.D. student at Departamento de Matemática, Universidade de Coimbra, supported by a scholarship from Fundação para a Ciência e a Tecnologia (FCT).

Support for J. Soares was provided by Centro de Matemática da Universidade de Coimbra.

Support for L.N. Vicente was provided by Centro de Matemática da Universidade de Coimbra and by FCT under grant POCI/59442/MAT/2004.

R. Silva · J. Soares $\cdot$ L.N. Vicente $(\bowtie)$

Departamento de Matemática, Universidade de Coimbra, 3001-454 Coimbra, Portugal

e-mail: lnv@mat.uc.pt

R. Silva

e-mail: renata@mat.uc.pt

J. Soares

e-mail: jsoares@mat.uc.pt 


\section{Introduction}

The local convergence theory of (infeasible) primal-dual interior-point methods for nonlinear programming was developed in the papers by El-Bakry et al. [5] and Yamashita and Yabe [14]. These papers show a q-quadratic rate of local convergence under the classical assumptions (second order sufficient optimality conditions, linear independence of the gradients of functions defining the binding constraints (LICQ), and strict complementarity). The study of q-superlinear convergence for quasi-Newton updates is reported in [10] and [14]. Furthermore, Vicente and Wright [13] proved a q-quadratic rate of convergence for a variant of the primal-dual interior-point method under degeneracy (replacing the LICQ by the Mangasarian-Fromowitz constraint qualification). In these approaches, the corresponding primal-dual interiorpoint method deals with the multipliers associated to both equality and inequality constraints as independent variables, and the primal-dual step is a Newton step for a perturbation of the first order necessary conditions for optimality. These approaches are infeasible since feasibility, corresponding to equality and, more importantly, to inequality constraints (rather than simple bounds), is only achieved asymptotically. Other rates of convergence for different interior-point methods for nonlinear programming have been established in [2, 3, 9], and [12].

Gould, Orban, Sartenaer, and Toint [7] investigated the rate of convergence of primal-dual logarithmic barrier interior-point methods for linear equality constraints and general inequalities. The log-barrier approach maintains the iterates strictly feasible with respect to the inequality constraints, and the multipliers corresponding to the equalities are treated implicitly as dependent variables. The authors proved q-superlinear convergence, with a rate that may be chosen arbitrarily close to quadratic. Basically, they studied conditions under which a single primal-dual Newton step is strictly feasible and satisfies appropriate log-barrier subproblem termination criteria.

The feasible primal-dual interior-point method of Tits et al. [11] achieves a quadratic rate of local convergence. In this algorithm, the multipliers corresponding to the inequality constraints are updated according to an appropriate formula and do not result directly from the Newton related primal-dual step on the perturbed KKT system of first-order optimality conditions. As we explain below, we are interested in analyzing the local convergence of a feasible primal-dual interior-point method without any special provision or correction formula for these multipliers.

In this paper we analyze the rate of local convergence of the feasible primal-dual interior-point method along the lines of the analyses in [5] and [14]. The aspect considered is that inequality constraints are not converted into equalities using slack variables. The method keeps strict feasibility with respect to the inequality constraints. The other components of the primal-dual interior-point method remain essentially the same: the primal-dual step is a Newton step on the perturbed KKT system and the various parameters are updated appropriately to induce a q-quadratic rate on the sequence of primal-dual iterates.

The material of this paper is organized in the following way. In Sect. 2, we describe the feasible primal-dual interior-point method in detail. The method is analyzed in Sect. 3 , where it is shown that the iterates converge locally with a q-quadratic rate in 
the case of concave inequalities. The analysis includes the case where the step length is computed inexactly. The nonconcave case is discussed in Sect. 4. The rate remains q-quadratic for nonconcave inequalities as long as the primal component of the step is asymptotically nonorthogonal to the gradients of the (nonconcave) functions defining the binding inequalities. In Sect. 5 we report some numerical results which show that the feasible method is competitive with the infeasible method in terms of number of iterations. We present a scheme to calculate the step length for the feasible method which requires a moderate number of constraint function evaluations. The paper is concluded in Sect. 6 with remarks about the theoretical interest and computational limitation of the analyzed approach.

\section{The feasible primal-dual interior-point method}

We consider the general nonlinear programming problem written in the form

$$
\begin{array}{ll}
\min & f(x), \\
\text { s.t. } & h(x)=0, \\
& g(x) \leq 0,
\end{array}
$$

where $f: \mathbb{R}^{n} \longrightarrow \mathbb{R}, h: \mathbb{R}^{n} \longrightarrow \mathbb{R}^{m_{h}}$, and $g: \mathbb{R}^{n} \longrightarrow \mathbb{R}^{m_{g}}$. The assumptions on the differentiability of the functions $f, g$, and $h$ will be stated later. The numbers $m_{h}$ and $m_{g}$ are assumed to be positive integers. The material of this paper remains valid in the case where there are no equality constraints $\left(m_{h}=0\right)$.

The Lagrangean function for problem (1) is $\ell: \mathbb{R}^{n+m_{h}+m_{g}} \longrightarrow \mathbb{R}$ defined by

$$
\ell(x, y, z)=f(x)+h(x)^{\top} y+g(x)^{\top} z,
$$

where $x$ are the primal variables and the pair $(y, z)$ represents the dual variables (or Lagrange multipliers). The gradient and the Hessian of $\ell$ with respect to the primal variables are given by

$$
\begin{aligned}
\nabla_{x} \ell(x, y, z) & =\nabla f(x)+\nabla h(x) y+\nabla g(x) z, \\
\nabla_{x x}^{2} \ell(x, y, z) & =\nabla^{2} f(x)+\sum_{j=1}^{m_{h}} y_{j} \nabla^{2} h_{j}(x)+\sum_{j=1}^{m_{g}} z_{j} \nabla^{2} g_{j}(x),
\end{aligned}
$$

whenever $f, g$, and $h$ are twice continuously differentiable at $x$.

The Karush-Kuhn-Tucker (KKT) first order (necessary optimality) conditions for problem (1) are described by

$$
\begin{aligned}
& F_{0}(x, y, z) \stackrel{\text { def }}{=}\left(\begin{array}{c}
\nabla_{x} \ell(x, y, z) \\
h(x) \\
-G(x) z
\end{array}\right)=0, \\
& g(x) \leq 0, \quad z \geq 0,
\end{aligned}
$$


where $G(x)=\operatorname{diag}(g(x))$. As we will see later, the primal-dual interior-point method is based on a perturbation of the conditions (2), given by

$$
\begin{aligned}
& F_{\mu}(x, y, z) \stackrel{\text { def }}{=}\left(\begin{array}{c}
\nabla_{x} \ell(x, y, z) \\
h(x) \\
-G(x) z-\mu e
\end{array}\right)=0, \\
& g(x)<0, \quad z>0,
\end{aligned}
$$

where $\mu$ is a positive scalar and $e$ is a vector of ones of dimension $m_{g}$. Note that, for $\bar{e}=\left(0,0, e^{\top}\right)^{\top} \in \mathbb{R}^{n+m_{h}+m_{g}}$,

$$
F_{\mu}(x, y, z)=F_{0}(x, y, z)-\mu \bar{e} .
$$

We will also make use of $\|\bar{e}\|=\|e\|$.

The main part of the iterative step of the primal-dual interior-point method consists of the linearization of the perturbed KKT system. One computes a primal-dual step $\Delta w=(\Delta x, \Delta y, \Delta z)$, by solving the linear system of equations

$$
F_{\mu}^{\prime}(w) \Delta w=-F_{\mu}(w)
$$

for fixed $w=(x, y, z)$ and $\mu>0$, where $F_{\mu}^{\prime}(w)$ is the Jacobian of $F_{\mu}(w)$. Notice that, from (3), $F_{\mu}^{\prime}(w)$ is also the Jacobian of $F_{0}(w)$. The primal-dual system (4) can be written by blocks in the form

$$
\left(\begin{array}{ccc}
\nabla_{x x}^{2} \ell(x, y, z) & \nabla h(x) & \nabla g(x) \\
\nabla h(x)^{\top} & 0 & 0 \\
-Z \nabla g(x)^{\top} & 0 & -G(x)
\end{array}\right)\left(\begin{array}{c}
\Delta x \\
\Delta y \\
\Delta z
\end{array}\right)=-\left(\begin{array}{c}
\nabla_{x} \ell(x, y, z) \\
h(x) \\
-G(x) z-\mu e
\end{array}\right),
$$

where $Z=\operatorname{diag}(z)$.

Most variants of the primal-dual interior-point method keep positive all the variables subject to nonnegativity constraints. In our case, it means keeping the multipliers $z$ positive. The parameter $\mu$ is driven to zero asymptotically. Since we are looking at the feasible variant of the primal-dual interior-point method, we must also keep $g(x)$ negative throughout the iterations. The main steps of this feasible variant are described below in Algorithm 2.1. For the purpose of analyzing local convergence, we do not include any stopping criterion.

Algorithm 2.1 (Feasible primal-dual interior-point method)

Choose an initial point $w_{0}=\left(x_{0}, y_{0}, z_{0}\right)$ with $g\left(x_{0}\right)<0$ and $z_{0}>0$.

For $k=0,1,2, \ldots$

Step 1. Choose the parameter $\mu_{k}>0$.

Step 2. Compute the solution $\Delta w_{k}=\left(\Delta x_{k}, \Delta y_{k}, \Delta z_{k}\right)$ of the system (5), for $x=x_{k}$, $y=y_{k}$, and $z=z_{k}$.

Step 3. Compute a positive step length $\alpha_{k}$ such that

$$
g\left(x_{k}+\alpha_{k} \Delta x_{k}\right)<0 \text { and } z_{k}+\alpha_{k} \Delta z_{k}>0 .
$$


Step 4. Define the next iterate $w_{k+1}=\left(x_{k+1}, y_{k+1}, z_{k+1}\right)$ according to:

$$
w_{k+1}=w_{k}+\alpha_{k} \Delta w_{k} .
$$

Since the step size $\alpha_{k}$ must satisfy (6) throughout the iterations, we will impose that

$$
\alpha_{k}=\min \left\{1, \tau_{k} \min _{i=1, \ldots, m_{g}}\left\{-\frac{\left(z_{k}\right)_{i}}{\left(\Delta z_{k}\right)_{i}}:\left(\Delta z_{k}\right)_{i}<0\right\}, \tau_{k} \min _{i=1, \ldots, m_{g}} \bar{\alpha}_{k}^{i}\right\},
$$

where $\tau_{k} \in(0,1)$ and

$$
\bar{\alpha}_{k}^{i} \leq \min \left\{\alpha: g_{i}\left(x_{k}+\alpha \Delta x_{k}\right)=0, \alpha>0\right\}, \quad i=1, \ldots, m_{g} .
$$

Whenever the minimum is not achieved, it is assumed by convention that it is set to $+\infty$.

We point out first that when the inequality constraints are of the simple bound type $(-x \leq 0)$, the choice for $\alpha_{k}$ is of the type given above with the inequalities in (9) satisfied as equalities. In general, when the functions defining the inequality constraints are nonlinear, it might be computationally expensive to actually determine the step lengths $\bar{\alpha}_{k}^{i}$ such that

$$
\bar{\alpha}_{k}^{i}=\min \left\{\alpha: g_{i}\left(x_{k}+\alpha \Delta x_{k}\right)=0, \alpha>0\right\}, \quad i=1, \ldots, m_{g} .
$$

On the other hand, to get a fast rate of local convergence one cannot compute step lengths $\bar{\alpha}_{k}^{i}$ that differ too much from (10). However, it is possible to allow a certain inexactness in this computation. Let us define the residuals

$$
r_{k}^{i} \stackrel{\text { def }}{=} g_{i}\left(x_{k}+\bar{\alpha}_{k}^{i} \Delta x_{k}\right), \quad i=1, \ldots, m_{g} .
$$

We will show that the feasible primal-dual interior-point method will retain local q-quadratic convergence as long as the residuals $r_{k}^{i}$ satisfy the condition

$$
-r_{k}^{i} \leq \min \left\{\sigma\left(-g_{i}\left(x_{k}\right)\right), \frac{-g_{i}\left(x_{k}\right) c_{1}\left\|\Delta w_{k}\right\|}{1+c_{1}\left\|\Delta w_{k}\right\|}\right\}, \quad i=1, \ldots, m_{g},
$$

where $\sigma \in(0,1)$ and $c_{1}>0$ are chosen independently of the iteration counter $k$. In Sect. 5.1 we will describe a scheme to compute $\bar{\alpha}_{k}^{i}$ that seems to be relatively efficient in practice.

Moreover, to achieve a q-quadratic rate of local convergence, the feasible primaldual interior-point method must update the parameters $\tau_{k} \in(0,1)$ and $\mu_{k}>0$ satisfying the classical conditions

$$
\begin{aligned}
1-\tau_{k} & \leq c_{2}\left\|F_{0}\left(w_{k}\right)\right\|, \\
\mu_{k} & \leq c_{3}\left\|F_{0}\left(w_{k}\right)\right\|^{2},
\end{aligned}
$$

where $c_{2}$ and $c_{3}$ are constants independent of $k$. Vector and matrix norms in this paper are chosen to be the Euclidean ones. 


\section{Analysis of local convergence}

The local convergence of the feasible primal-dual interior-point method is analyzed at a point $x_{*}$ satisfying the following assumptions. In what follows, $B\left(a_{*} ; r\right)$ denotes the open ball $\left\{a \in \mathbb{R}^{d}:\left\|a-a_{*}\right\|<r\right\}$ of radius $r$ centered at $a_{*}$.

(A1) There exists an $\epsilon>0$ such that the functions $f, g$, and $h$ are twice continuously differentiable in the ball $B\left(x_{*} ; \epsilon\right)$. Moreover, the second order partial derivatives of $f, g$, and $h$ are Lipschitz continuous in $B\left(x_{*} ; \epsilon\right)$.

(A2) The point $x_{*}$ is feasible and the gradients of the active constraints are linearly independent at $x_{*}$.

(A3) There exist Lagrange multipliers $y_{*}$ and $z_{*}$ such that $w_{*}=\left(x_{*}, y_{*}, z_{*}\right)$ satisfies the first order KKT conditions and the second order sufficient conditions and such that the pair $\left(-g\left(x_{*}\right), z_{*}\right)$ satisfies the strict complementarity condition $\left(-g\left(x_{*}\right)+z_{*}>0\right)$.

Assumptions A1-A3 are the classical (nondegenerate) assumptions used to locally analyze interior-point methods. It results from Assumption A3 that the multipliers associated with the inequalities are nonnegative $\left(z_{*} \geq 0\right)$ and also that

$$
F_{0}\left(w_{*}\right)=0
$$

We recall now the basic smoothness results that are required in the proof of the local convergence of the primal-dual interior-point method.

Lemma 3.1 Let $x_{*}$ be a point for which Assumptions A1-A3 hold and $w_{*}=$ $\left(x_{*}, y_{*}, z_{*}\right)$. Then, there exists a positive constant $\gamma$ such that

$$
\begin{aligned}
\left\|F_{0}\left(w^{1}\right)-F_{0}\left(w^{2}\right)\right\| & \leq \gamma\left\|w^{1}-w^{2}\right\|, \\
\left\|F_{0}^{\prime}\left(w^{1}\right)-F_{0}^{\prime}\left(w^{2}\right)\right\| & \leq \gamma\left\|w^{1}-w^{2}\right\|, \\
\left\|F_{0}\left(w^{1}\right)-F_{0}\left(w^{2}\right)-F_{0}^{\prime}\left(w^{2}\right)\left(w^{1}-w^{2}\right)\right\| & \leq \frac{1}{2} \gamma\left\|w^{1}-w^{2}\right\|^{2},
\end{aligned}
$$

for all $w^{1}$ and $w^{2}$ in $B\left(w_{*} ; \epsilon\right)$.

The next lemma states that the primal-dual matrix is nonsingular around $w_{*}$, in the sense that is of interest to us. For a proof see, for instance, [6].

Lemma 3.2 Let $x_{*}$ be a point for which Assumptions A1-A3 hold and $w_{*}=$ $\left(x_{*}, y_{*}, z_{*}\right)$. Then the following holds:

(i) $F_{0}^{\prime}\left(w_{*}\right)$ is nonsingular;

(ii) $F_{0}^{\prime}(w)$ is nonsingular for $w$ in $B\left(w_{*} ; \epsilon_{n s}\right)$, for some $\epsilon_{n s}$ satisfying $0<\epsilon_{n s}<\epsilon$.

From this lemma, it is assured the existence of a constant $\zeta>0$ such that

$$
\left\|F_{0}^{\prime}(w)^{-1}\right\|=\left\|F_{\mu}^{\prime}(w)^{-1}\right\| \leq \zeta
$$


for all $w$ in $B\left(w_{*} ; \epsilon_{n s}\right)$. For such points $w$, the primal-dual step $\Delta w$ given by the solution of the system (4) is well-defined and is equal to

$$
\Delta w=-F_{\mu}^{\prime}(w)^{-1} F_{\mu}(w) .
$$

The local asymptotic behavior of the feasible primal-dual interior-point method is studied first for concave binding inequalities.

(A4) The functions $g_{i}$, for $i \in\left\{1, \ldots, m_{g}\right\}$ such that $g_{i}\left(x_{*}\right)=0$, are concave.

The main part of the analysis is spent proving a lower bound for the length of the step size parameter $\alpha_{k}$.

Lemma 3.3 Let $x_{*}$ be a point for which Assumptions A1-A4 hold and $w_{*}=$ $\left(x_{*}, y_{*}, z_{*}\right)$. Consider a sequence $\left\{w_{k}=\left(x_{k}, y_{k}, z_{k}\right)\right\}$ generated by the feasible primal-dual interior-point method described in Algorithm 2.1. If $\alpha_{k}$ satisfies (8-9) and (11) and $\tau_{k} \in(0,1)$ and $\mu_{k}>0$ satisfy (12) and (13), then there exist positive constants $\varepsilon$ and $\kappa$ independent of $k$ such that, when

$$
w_{k} \in B\left(w_{*} ; \varepsilon\right)
$$

either $\alpha_{k}=1$ or the bound

$$
1-\alpha_{k} \leq\left(1-\tau_{k}\right)+\kappa \zeta\left(\left\|F_{0}\left(w_{k}\right)\right\|+\mu_{k}\|e\|\right)
$$

holds for all iterates $k$.

Proof First we have to set $\varepsilon=\epsilon_{n s}$, where $\epsilon_{n s}$ is given in Lemma 3.2.

Using (18), (3), (14), (17), (15), and (19) sequentially, it is easily derived the following bound for the primal-dual step:

$$
\begin{aligned}
\left\|\Delta w_{k}\right\| & =\left\|F_{\mu}^{\prime}\left(w_{k}\right)^{-1} F_{\mu}\left(w_{k}\right)\right\| \\
& \leq\left\|F_{\mu}^{\prime}\left(w_{k}\right)^{-1}\right\|\left(\left\|F_{0}\left(w_{k}\right)\right\|+\mu_{k}\|e\|\right) \\
& \leq \zeta\left(\gamma\left\|w_{k}-w^{*}\right\|+\mu_{k}\|e\|\right) \\
& \leq \zeta\left(\gamma \varepsilon+\mu_{k}\|e\|\right) .
\end{aligned}
$$

Thus, from the condition (13) on the size of $\mu_{k}$, and given a constant $\eta>0$, one can reduce $\varepsilon$ if necessary such that

$$
\left\|\Delta w_{k}\right\| \leq \eta
$$

In particular, it is possible to choose a sufficiently small $\varepsilon$ such that

$$
\kappa\left\|\Delta w_{k}\right\| \leq \tau_{k}
$$

where $\kappa$ is defined by

$$
\kappa \stackrel{\text { def }}{=} \max \left\{\frac{\kappa_{2}}{1-\sigma}, \kappa_{1}+\kappa_{1} c_{1} \eta+c_{1}\right\} .
$$


The constants $\kappa_{1}$ and $\kappa_{2}$ are given by

$$
\kappa_{1}=2 \max \left\{\frac{1}{\left(z_{*}\right)_{i}}:\left(z_{*}\right)_{i}>0, i \in\left\{1, \ldots, m_{g}\right\}\right\}
$$

and

$$
\kappa_{2}=2 M_{\nabla g} \max \left\{-\frac{1}{g_{i}\left(x_{*}\right)}: g_{i}\left(x_{*}\right)<0, i \in\left\{1, \ldots, m_{g}\right\}\right\},
$$

where $M_{\nabla g}$ is an upper bound on the size of $\nabla g$ in $B\left(x_{*} ; \epsilon\right)$.

We divide the proof in two separate cases: the case where the step length is defined by a multiplier and the case where the step length is defined by an inequality.

Case where step length is defined by a multiplier. In this first case we assume that there exists an index $i \in\left\{1, \ldots, m_{g}\right\}$ for which $\left(\Delta z_{k}\right)_{i}<0$ and

$$
\alpha_{k}=-\tau_{k} \frac{\left(z_{k}\right)_{i}}{\left(\Delta z_{k}\right)_{i}} .
$$

If $i$ is such that $\left(z_{*}\right)_{i}>0$ then, from the definition of $\kappa$ and from (22),

$$
\alpha_{k}=\tau_{k} \frac{\left(z_{k}\right)_{i}}{-\left(\Delta z_{k}\right)_{i}} \geq \frac{\tau_{k}}{\kappa\left\|\Delta w_{k}\right\|} \geq 1 .
$$

When $\left(z_{*}\right)_{i}=0$ (and $g_{i}\left(x_{*}\right)<0$ ), we make use of the primal-dual block equation (see (5))

$$
-Z_{k} \nabla g\left(x_{k}\right)^{\top} \Delta x_{k}-G\left(x_{k}\right) \Delta z_{k}=G\left(x_{k}\right) z_{k}+\mu_{k} e
$$

to write

$$
-\left(z_{k}\right)_{i} \nabla g_{i}\left(x_{k}\right)^{\top} \Delta x_{k}-g_{i}\left(x_{k}\right)\left(\Delta z_{k}\right)_{i}=g_{i}\left(x_{k}\right)\left(z_{k}\right)_{i}+\mu_{k},
$$

or equivalently,

$$
-\frac{\left(\Delta z_{k}\right)_{i}}{\left(z_{k}\right)_{i}}=1+\frac{\mu_{k}}{g_{i}\left(x_{k}\right)\left(z_{k}\right)_{i}}+p_{k}^{i}
$$

with

$$
p_{k}^{i}=\frac{\nabla g_{i}\left(x_{k}\right)^{\top} \Delta x_{k}}{g_{i}\left(x_{k}\right)} \leq \frac{\left|\nabla g_{i}\left(x_{k}\right)^{\top} \Delta x_{k}\right|}{-g_{i}\left(x_{k}\right)} \leq \kappa\left\|\Delta w_{k}\right\| .
$$

Thus, since $\mu_{k} /\left(g_{i}\left(x_{k}\right)\left(z_{k}\right)_{i}\right)<0$,

$$
-\frac{\left(\Delta z_{k}\right)_{i}}{\left(z_{k}\right)_{i}} \leq 1+\kappa\left\|\Delta w_{k}\right\|
$$

and

$$
\alpha_{k}=\tau_{k} \frac{\left(z_{k}\right)_{i}}{-\left(\Delta z_{k}\right)_{i}} \geq \frac{\tau_{k}}{1+\kappa\left\|\Delta w_{k}\right\|} \geq \tau_{k}\left(1-\kappa\left\|\Delta w_{k}\right\|\right) .
$$

Case where step length is defined by an inequality. Now we are interested in the case

$$
\alpha_{k}=\tau_{k} \bar{\alpha}_{k}^{i}
$$


for some index $i \in\left\{1, \ldots, m_{g}\right\}$. By applying the mean value theorem, we have

$$
r_{k}^{i}-g_{i}\left(x_{k}\right)=g_{i}\left(x_{k}+\bar{\alpha}_{k}^{i} \Delta x_{k}\right)-g_{i}\left(x_{k}\right)=\bar{\alpha}_{k}^{i} \nabla g_{i}\left(x_{k}+t_{k}^{i} \bar{\alpha}_{k}^{i} \Delta x_{k}\right)^{\top} \Delta x_{k},
$$

for some $t_{k}^{i} \in(0,1)$, and the step length $\bar{\alpha}_{k}^{i}$ can be written as

$$
\bar{\alpha}_{k}^{i}=\frac{r_{k}^{i}-g_{i}\left(x_{k}\right)}{\nabla g_{i}\left(x_{k}+t_{k}^{i} \bar{\alpha}_{k}^{i} \Delta x_{k}\right)^{\top} \Delta x_{k}} .
$$

Since $-r_{k}^{i} \leq \sigma\left(-g_{i}\left(x_{k}\right)\right)$, both the numerator and the denominator in this expression for $\bar{\alpha}_{k}^{i}$ are positive.

If $i$ is such that $g_{i}\left(x_{*}\right)<0$ then, from the definitions of $\kappa_{2}$ and $\kappa$ and from (22),

$$
\begin{aligned}
\alpha_{k}=\tau_{k} \bar{\alpha}_{k}^{i} & \geq \tau_{k} \frac{(1-\sigma)\left(-g_{i}\left(x_{k}\right)\right)}{\nabla g_{i}\left(x_{k}+t_{k}^{i} \bar{\alpha}_{k}^{i} \Delta x_{k}\right)^{\top} \Delta x_{k}} \\
& \geq \tau_{k} \frac{(1-\sigma)\left(-g_{i}\left(x_{k}\right)\right)}{\left\|\nabla g_{i}\left(x_{k}+t_{k}^{i} \bar{\alpha}_{k}^{i} \Delta x_{k}\right)\right\|\left\|\Delta x_{k}\right\|} \\
& \geq \tau_{k} \frac{(1-\sigma)}{\kappa_{2}\left\|\Delta w_{k}\right\|} \\
& \geq \frac{\tau_{k}}{\kappa\left\|\Delta w_{k}\right\|} \\
& \geq 1 .
\end{aligned}
$$

When $g_{i}\left(x_{*}\right)=0$ (and $\left(z_{*}\right)_{i}>0$ ), we must first add and subtract

$$
r_{k}^{i} \frac{\left(\Delta z_{k}\right)_{i}}{\left(z_{k}\right)_{i}}+r_{k}^{i}+r_{k}^{i} \frac{\mu_{k}}{g_{i}\left(x_{k}\right)\left(z_{k}\right)_{i}}
$$

to the right hand side in the primal-dual equation

$$
-\nabla g_{i}\left(x_{k}\right)^{\top} \Delta x_{k}=g_{i}\left(x_{k}\right) \frac{\left(\Delta z_{k}\right)_{i}}{\left(z_{k}\right)_{i}}+g_{i}\left(x_{k}\right)+g_{i}\left(x_{k}\right) \frac{\mu_{k}}{g_{i}\left(x_{k}\right)\left(z_{k}\right)_{i}} .
$$

After division by $g_{i}\left(x_{k}\right)-r_{k}^{i}$, this results in

$$
\begin{aligned}
-\frac{\nabla g_{i}\left(x_{k}\right)^{\top} \Delta x_{k}}{g_{i}\left(x_{k}\right)-r_{k}^{i}}= & \frac{\left(\Delta z_{k}\right)_{i}}{\left(z_{k}\right)_{i}}+1+\frac{\mu_{k}}{g_{i}\left(x_{k}\right)\left(z_{k}\right)_{i}} \\
& +\frac{r_{k}^{i}}{g_{i}\left(x_{k}\right)-r_{k}^{i}} \frac{\left(\Delta z_{k}\right)_{i}}{\left(z_{k}\right)_{i}}+\frac{r_{k}^{i}}{g_{i}\left(x_{k}\right)-r_{k}^{i}}+\frac{\mu_{k} r_{k}^{i}}{g_{i}\left(x_{k}\right)\left(z_{k}\right)_{i}\left(g_{i}\left(x_{k}\right)-r_{k}^{i}\right)} .
\end{aligned}
$$

Since the third and the sixth terms in the right hand side of this equality are negative and since, from (11),

$$
\frac{r_{k}^{i}}{g_{i}\left(x_{k}\right)-r_{k}^{i}} \leq c_{1}\left\|\Delta w_{k}\right\|
$$


we obtain, from (21),

$$
\begin{aligned}
-\frac{\nabla g_{i}\left(x_{k}\right)^{\top} \Delta x_{k}}{g_{i}\left(x_{k}\right)-r_{k}^{i}} & \leq 1+\kappa_{1}\left\|\Delta w_{k}\right\|+\kappa_{1} c_{1}\left\|\Delta w_{k}\right\|^{2}+c_{1}\left\|\Delta w_{k}\right\| \\
& \leq 1+\left(\kappa_{1}+\kappa_{1} c_{1} \eta+c_{1}\right)\left\|\Delta w_{k}\right\| \\
& \leq 1+\kappa\left\|\Delta w_{k}\right\| .
\end{aligned}
$$

Now, from the concavity of $g_{i}$, we derive

$$
\begin{aligned}
- & \frac{\nabla g_{i}\left(x_{k}+t_{k}^{i} \bar{\alpha}_{k}^{i} \Delta x_{k}\right)^{\top} \Delta x_{k}}{g_{i}\left(x_{k}\right)-r_{k}^{i}} \\
& =-\frac{\nabla g_{i}\left(x_{k}+t_{k}^{i} \bar{\alpha}_{i} \Delta x_{k}\right)^{\top} \Delta x_{k}}{g_{i}\left(x_{k}\right)-r_{k}^{i}}+\frac{\nabla g_{i}\left(x_{k}\right)^{\top} \Delta x_{k}}{g_{i}\left(x_{k}\right)-r_{k}^{i}}-\frac{\nabla g_{i}\left(x_{k}\right)^{\top} \Delta x_{k}}{g_{i}\left(x_{k}\right)-r_{k}^{i}} \\
& =\frac{\left[\nabla g_{i}\left(x_{k}+t_{k}^{i} \bar{\alpha}_{k}^{i} \Delta x_{k}\right)-\nabla g_{i}\left(x_{k}\right)\right]^{\top} \Delta x_{k}}{r_{k}^{i}-g_{i}\left(x_{k}\right)}-\frac{\nabla g_{i}\left(x_{k}\right)^{\top} \Delta x_{k}}{g_{i}\left(x_{k}\right)-r_{k}^{i}} \\
& \leq-\frac{\nabla g_{i}\left(x_{k}\right)^{\top} \Delta x_{k}}{g_{i}\left(x_{k}\right)-r_{k}^{i}} \\
& \leq 1+\kappa\left\|\Delta w_{k}\right\|
\end{aligned}
$$

and

$$
\alpha_{k}=\tau_{k} \bar{\alpha}_{k}^{i}=\tau_{k} \frac{r_{k}^{i}-g_{i}\left(x_{k}\right)}{\nabla g_{i}\left(x_{k}+t_{k}^{i} \bar{\alpha}_{k}^{i} \Delta x_{k}\right)^{\top} \Delta x_{k}} \geq \frac{\tau_{k}}{1+\kappa\left\|\Delta w_{k}\right\|} \geq \tau_{k}\left(1-\kappa\left\|\Delta w_{k}\right\|\right) .
$$

Conclusion. Combining all the four bounds derived for $\alpha_{k}$ (two in each case considered), one obtains

$$
\alpha_{k} \geq \min \left\{1, \tau_{k}\left(1-\kappa\left\|\Delta w_{k}\right\|\right)\right\}=\tau_{k}\left(1-\kappa\left\|\Delta w_{k}\right\|\right) \geq \tau_{k}-\kappa\left\|\Delta w_{k}\right\| .
$$

The last inequality above is based on the fact that $\tau_{k}<1$ for all $k$ provided $\varepsilon$ is chosen small enough. Finally, from this lower bound on $\alpha_{k}$, we get

$$
0 \leq 1-\alpha_{k} \leq\left(1-\tau_{k}\right)+\kappa\left\|\Delta w_{k}\right\| \leq\left(1-\tau_{k}\right)+\kappa \zeta\left(\left\|F_{0}\left(w_{k}\right)\right\|+\mu_{k}\|e\|\right),
$$

which concludes the proof of the lemma.

We can state now the q-quadratic rate of local convergence of Algorithm 2.1. The proof can be found in [14] and we describe it briefly for completeness.

Theorem 3.1 Let $x_{*}$ be a point for which Assumptions A1-A4 hold and $w_{*}=$ $\left(x_{*}, y_{*}, z_{*}\right)$. Consider a sequence $\left\{w_{k}=\left(x_{k}, y_{k}, z_{k}\right)\right\}$ generated by the feasible primal-dual interior-point method described in Algorithm 2.1. If $\alpha_{k}$ satisfies (8-9) 
and (11) and $\tau_{k} \in(0,1)$ and $\mu_{k}>0$ satisfy (12) and (13), then there exists a positive constant $\varepsilon$ independent of $k$ such that, when

$$
w_{0} \in B\left(w_{*} ; \varepsilon\right),
$$

the sequence $\left\{w_{k}\right\}$ is well defined and converges to $w_{*}$. Moreover, we have

$$
\left\|w_{k+1}-w_{*}\right\| \leq v\left\|w_{k}-w_{*}\right\|^{2}
$$

for all iterates $k$, where $v$ is a positive constant independent of $k$.

Proof Let us assume that $\left\|w_{k}-w_{*}\right\|<\varepsilon$. By applying (7), (18), (3), and (14), we obtain

$$
\begin{aligned}
w_{k+1}-w_{*}= & \left(w_{k}-w_{*}\right)+\alpha_{k} \Delta w_{k} \\
= & \left(1-\alpha_{k}\right)\left(w_{k}-w_{*}\right) \\
& +\alpha_{k} F_{\mu_{k}}^{\prime}\left(w_{k}\right)^{-1}\left[F_{0}\left(w_{*}\right)-F_{0}\left(w_{k}\right)-F_{\mu_{k}}^{\prime}\left(w_{k}\right)\left(w_{*}-w_{k}\right)+\mu_{k} \bar{e}\right] .
\end{aligned}
$$

Now, using (20), (16), (17), and $\alpha_{k} \leq 1$, we have, for a sufficiently small $\varepsilon$,

$$
\begin{aligned}
\left\|w_{k+1}-w_{*}\right\| \leq & \left(1-\alpha_{k}\right)\left\|w_{k}-w_{*}\right\| \\
& +\alpha_{k}\left\|F_{\mu_{k}}^{\prime}\left(w_{k}\right)^{-1}\right\|\left\|F_{0}\left(w_{*}\right)-F_{0}\left(w_{k}\right)-F_{\mu_{k}}^{\prime}\left(w_{k}\right)\left(w_{*}-w_{k}\right)\right\| \\
& +\alpha_{k} \mu_{k}\left\|F_{\mu_{k}}^{\prime}\left(w_{k}\right)^{-1}\right\|\|e\| \\
\leq & {\left[\left(1-\tau_{k}\right)+\kappa \zeta\left\|F_{0}\left(w_{k}\right)\right\|+\kappa \zeta\|e\| \mu_{k}\right]\left\|w_{k}-w_{*}\right\| } \\
& +\frac{\zeta \gamma}{2}\left\|w_{k}-w_{*}\right\|^{2}+\|e\| \zeta \mu_{k} .
\end{aligned}
$$

We also known that $\tau_{k}$ and $\mu_{k}$ satisfy (12) and (13). Thus, using the fact that $\left\|F_{0}\left(w_{k}\right)\right\|=\left\|F_{0}\left(w_{k}\right)-F_{0}\left(w_{*}\right)\right\| \leq \gamma\left\|w_{k}-w_{*}\right\|$, we assure the existence of a constant $v>0$ independent of the iterates such that (26) holds. It is possible to prove by induction that $\left\{w_{k}\right\}$ converges to $w_{*}$, if $\varepsilon$ is chosen sufficiently small. The inequality (26) shows that the local convergence rate is q-quadratic.

\section{The nonconcave case}

The concavity of the inequality constraint functions was required in (25) when the binding constraint function $g_{i}$ was responsible for the step size $\alpha_{k}$. However, one can see that the method retains a q-quadratic rate in the nonconcave case as long as there exists a positive constant $\beta$ such that

$$
r_{k}^{i}-g_{i}\left(x_{k}\right)=g_{i}\left(x_{k}+\bar{\alpha}_{k}^{i} \Delta x_{k}\right)-g_{i}\left(x_{k}\right) \geq \beta \bar{\alpha}_{k}^{i}\left\|\Delta x_{k}\right\|
$$

for all $k$ and all indices $i$ corresponding to $g_{i}\left(x_{*}\right)=0$. In fact, one would get

$$
\frac{\left[\nabla g_{i}\left(x_{k}+t_{k}^{i} \bar{\alpha}_{k}^{i} \Delta x_{k}\right)-\nabla g_{i}\left(x_{k}\right)\right]^{\top} \Delta x_{k}}{r_{k}^{i}-g_{i}\left(x_{k}\right)} \leq \frac{L \nabla g_{i}}{\beta}\left\|\Delta x_{k}\right\|,
$$


where $L_{\nabla g_{i}}$ is the Lipschitz constant of the function $\nabla g_{i}$ in $B\left(x_{*} ; \epsilon\right)$. Then, from (23) and (24),

$$
\begin{aligned}
\frac{1}{\bar{\alpha}_{k}^{i}}=-\frac{\nabla g_{i}\left(x_{k}+t_{k}^{i} \bar{\alpha}_{k}^{i} \Delta x_{k}\right)^{\top} \Delta x_{k}}{g_{i}\left(x_{k}\right)-r_{k}^{i}} & \left.\leq 1+\left[\left(\kappa_{1}+\kappa_{1} c_{1} \eta+c_{1}\right)+L_{\nabla g_{i}} / \beta\right)\right]\left\|\Delta w_{k}\right\| \\
& \leq 1+\kappa\left\|\Delta w_{k}\right\|,
\end{aligned}
$$

after an appropriate redefinition of $\kappa$.

The bound (27) is satisfied for $k$ sufficiently large as long as

$$
\liminf _{k \longrightarrow+\infty} \nabla g_{i}\left(x_{k}\right)^{\top} \frac{\Delta x_{k}}{\left\|\Delta x_{k}\right\|}=4 \beta>0
$$

To see why this is true let us expand $g_{i}\left(x_{k}+\bar{\alpha}_{k}^{i} \Delta x_{k}\right)$ around $x_{k}$ :

$g_{i}\left(x_{k}+\bar{\alpha}_{k}^{i} \Delta x_{k}\right)-g_{i}\left(x_{k}\right)=\bar{\alpha}_{k}^{i} \nabla g_{i}\left(x_{k}\right)^{\top} \Delta x_{k}+\frac{\left(\bar{\alpha}_{k}^{i}\right)^{2}}{2} \Delta x_{k}^{\top} \nabla^{2} g_{i}\left(x_{k}+s_{k}^{i} \bar{\alpha}_{k}^{i} \Delta x_{k}\right) \Delta x_{k}$,

for some $s_{k}^{i} \in(0,1)$. Since we are only looking at the cases where $\bar{\alpha}_{k}^{i} \leq 1$, one can see that

$$
\frac{g_{i}\left(x_{k}+\bar{\alpha}_{k}^{i} \Delta x_{k}\right)-g_{i}\left(x_{k}\right)}{\bar{\alpha}_{k}^{i}\left\|\Delta x_{k}\right\|} \geq \beta
$$

holds for $k$ sufficiently large as long as

$$
\left\|\Delta x_{k}\right\| \leq\left\|\Delta w_{k}\right\| \leq \frac{2 \beta}{M_{\nabla^{2} g_{i}}}
$$

where $M_{\nabla^{2} g_{i}}$ is an upper bound on the size of the Hessian $\nabla^{2} g_{i}$ in $B\left(x_{*} ; \epsilon\right)$, requiring again a redefinition of $\kappa$.

Condition (28) has no influence if the constraint is concave because, even when this condition is not satisfied, the concavity of the function defining the constraint allows a locally full step $\left(\alpha_{k}=1\right)$ with respect to that constraint.

\section{Implementation and numerical results}

We have implemented Algorithm 2.1 (the feasible primal-dual interior point method) and tested it on a set of small CUTEr problems [8]. Subsection 5.1 completes the algorithm description, namely, we explain there how to compute, without too much effort, a stepsize that satisfies condition (11). As a baseline for comparison, we have also tested the infeasible primal-dual interior point method (details are explained in Subsect. 5.2). Numerical results are reported in Subsect. 5.3. 


\subsection{Step size calculation for the feasible method}

To achieve a q-quadratic rate of convergence, condition (11) is imposed on the size of the residual $r_{k}^{i}=g_{i}\left(x_{k}+\bar{\alpha}_{k}^{i} \Delta x_{k}\right)$. A simple procedure to calculate $\bar{\alpha}_{k}^{i}$ is Newton's method, fully described in Algorithm 5.1 below. Obviously, the scheme is to be applied only to the nonlinear inequality constraints, as for the linear constraints (including bounds) one can easily determine the exact maximum allowed step length.

Algorithm 5.1 (Step size calculation for the feasible method)

Choose an initial step size $\alpha>0$ (for instance, $\alpha=0.5$ if $k=0$ and $\alpha=\bar{\alpha}_{k-1}^{i}$ for $k \geq 1$ ). Choose $\sigma \in(0,1)$ and $c_{1}>0$ (for instance, $\sigma=10^{-2}$ and $c_{1}=0.5$ ). Set also $\Delta \alpha=1$.

While $|\Delta \alpha|>10^{-3}$ and $-g_{i}\left(x_{k}+\alpha \Delta x_{k}\right)>\min \left\{\sigma\left(-g_{i}\left(x_{k}\right)\right), \frac{-g_{i}\left(x_{k}\right) c_{1}\left\|\Delta w_{k}\right\|}{1+c_{1}\left\|\Delta w_{k}\right\|}\right\}$ do

$$
\text { compute } \Delta \alpha=-\frac{g_{i}\left(x_{k}+\alpha \Delta x_{k}\right)}{\nabla g_{i}\left(x_{k}+\alpha \Delta x_{k}\right)^{\top} \Delta x_{k}} \text { and replace } \alpha \text { by } \alpha+\Delta \alpha \text {. }
$$

After termination set $\bar{\alpha}_{k}^{i}=\alpha$.

We incorporated a safeguard for negative values of $\alpha$. When $\alpha$ became negative we projected it back to the positive axis by setting it to $10^{-2}$. This safeguard acted only in two of the problems tested (leading then to a convergent run).

We also experimented an alternative scheme where the calculation of the derivatives $\nabla g_{i}\left(x_{k}+\alpha \Delta x_{k}\right)^{\top} \Delta x_{k}$ was approximated by $\nabla g_{i}\left(x_{k}\right)^{\top} \Delta x_{k}$. While this alternative seemed not to affect the efficiency of the feasible method (in terms of number of iterations), it provided a less robust method (in the sense that less problems were solved).

\subsection{The infeasible primal-dual interior-point method}

As a baseline for comparison we also ran the infeasible primal-dual interior-point method reported in Algorithm 5.2. This version of the infeasible method is the same as the feasible method applied to a reformulated problem, where inequality constraints are converted into equalities with the introduction of nonnegative slack variables. The feasible region can thus be written as $\left\{\hat{x} \in \mathbb{R}^{\hat{n}}: \hat{h}(\hat{x})=0, \hat{x} \geq 0\right\}$ and the Lagrangean function as $\hat{\ell}(\hat{x}, \hat{y}, \hat{z})=\hat{f}(\hat{x})+\hat{h}(\hat{x})^{\top} \hat{y}-\hat{x}^{\top} \hat{z}$.

Algorithm 5.2 (Infeasible primal-dual interior-point method)

Choose an initial point $w_{0}=\left(\hat{x}_{0}, \hat{y}_{0}, \hat{z}_{0}\right)$ with $\hat{x}_{0}>0$ and $\hat{z}_{0}>0$.

For $k=0,1,2, \ldots$

Step 1. Choose the parameter $\mu_{k}>0$.

Step 2. Compute the solution $\Delta w_{k}=\left(\Delta \hat{x}_{k}, \Delta \hat{y}_{k}, \Delta \hat{z}_{k}\right)$ of the system

$$
\left(\begin{array}{ccc}
\nabla_{\hat{x} \hat{x}}^{2} \hat{\ell}\left(\hat{x}_{k}, \hat{y}_{k}, \hat{z}_{k}\right) & \nabla \hat{h}\left(\hat{x}_{k}\right) & -I_{\hat{n} \times \hat{n}} \\
\nabla \hat{h}\left(\hat{x}_{k}\right)^{\top} & 0 & 0 \\
\hat{Z}_{k} & 0 & \hat{X}_{k}
\end{array}\right)\left(\begin{array}{c}
\Delta \hat{x}_{k} \\
\Delta \hat{y}_{k} \\
\Delta \hat{z}_{k}
\end{array}\right)=-\left(\begin{array}{c}
\nabla_{\hat{x}} \hat{\ell}\left(\hat{x}_{k}, \hat{y}_{k}, \hat{z}_{k}\right) \\
\hat{h}\left(\hat{x}_{k}\right) \\
\hat{X}_{k} \hat{z}_{k}-\mu_{k} e_{\hat{n}}
\end{array}\right),
$$

where $\hat{X}_{k}=\operatorname{diag}\left(\hat{x}_{k}\right)$ and $\hat{Z}_{k}=\operatorname{diag}\left(\hat{z}_{k}\right)$. 
Step 3. Choose $\tau_{k} \in(0,1)$. Compute a positive step length $\alpha_{k}$ such that

$$
\begin{gathered}
\alpha_{k}=\min \left\{1, \tau_{k} \min _{i=1, \ldots, \hat{n}}\left\{-\frac{\left(\hat{x}_{k}\right)_{i}}{\left(\Delta \hat{x}_{k}\right)_{i}}:\left(\Delta \hat{x}_{k}\right)_{i}<0\right\},\right. \\
\left.\tau_{k} \min _{i=1, \ldots, \hat{n}}\left\{-\frac{\left(\hat{z}_{k}\right)_{i}}{\left(\Delta \hat{z}_{k}\right)_{i}}:\left(\Delta \hat{z}_{k}\right)_{i}<0\right\}\right\} .
\end{gathered}
$$

Step 4. Define the next iterate $w_{k+1}=\left(\hat{x}_{k+1}, \hat{y}_{k+1}, \hat{z}_{k+1}\right)$ according to:

$$
w_{k+1}=w_{k}+\alpha_{k} \Delta w_{k}
$$

\subsection{Numerical results}

We tested both feasible and infeasible primal-dual interior point methods (Algorithms 2.1 and 5.2) on a set of small CUTEr [8] problems. We restricted our attention to problems with nonlinear inequality constraints and for which the initial point $x_{0}$, provided by CUTEr, satisfies $g\left(x_{0}\right)<0$.

The initial primal point given to the infeasible method was the point $x_{0}$ provided by CUTEr. When the initial primal values are nonpositive we projected them to the positive axis. In some cases, not all variables are restricted in sign and the infeasible method was adapted to take care of this situation. The remaining components of the initial vector for the infeasible method are defined as follows: $\hat{y}_{0}=\operatorname{argmin}_{\hat{y}}\left\|\nabla \hat{h}\left(\hat{x}_{0}\right) \hat{y}+\left[-\hat{z}_{0}^{i n}+\nabla \hat{f}\left(\hat{x}_{0}\right)\right]\right\|$ and $\hat{z}_{0}=\max \left\{\nabla \hat{h}\left(\hat{x}_{0}\right) \hat{y}_{0}+\right.$ $\left.\nabla \hat{f}\left(\hat{x}_{0}\right), \hat{z}_{0}^{i n}\right\}$, where $\hat{z}_{0}^{i n}$ is a vector of ones of the appropriate size. For the feasible method, $x_{0}$ is provided by CUTEr (satisfying $g\left(x_{0}\right)<0$ ). Then, we compute $y_{0}=$ $\operatorname{argmin}_{y}\left\|\nabla h\left(x_{0}\right) y+\left[\nabla g\left(x_{0}\right) z_{0}^{i n}+\nabla f\left(x_{0}\right)\right]\right\|$ and $z_{0}=\max _{2}\left\{\operatorname{argmin}_{z} \| \nabla g\left(x_{0}\right) z+\right.$ $\left.\left[\nabla h\left(x_{0}\right) y_{0}+\nabla f\left(x_{0}\right)\right] \|, z_{0}^{i n}\right\}$.

The codes were implemented in Fortran 90 and ran on a Compaq Tru64 (operating system Unix V5.1, 2 GB RAM, Alpha 21264A 667 MHz). For both algorithms, we updated $\tau_{k}$ and $\mu_{k}$ as follows:

$$
1-\tau_{k}=\min \left\{10^{-2}, 10^{-2}\left\|F_{0}\left(w_{k}\right)\right\|\right\} \quad \text { and } \quad \mu_{k}=\min \left\{10^{-2}, 10^{-1}\left\|F_{0}\left(w_{k}\right)\right\|^{2}\right\} .
$$

The stopping criterion was $\left\|F_{0}\left(w_{k}\right)\right\| \leq 10^{-8}$ for the feasible method and $\left\|\left(\nabla_{\hat{x}} \hat{\ell}\left(\hat{x}_{k}, \hat{y}_{k}, \hat{z}_{k}\right), \hat{h}\left(\hat{x}_{k}\right), \hat{X}_{k} \hat{z}_{k}\right)\right\| \leq 10^{-8}$ for the infeasible case. The linear algebra was implemented in the dense form using the LAPACK [1] routines DGESV (for the primal-dual systems) and DGELSD (for the least-squares multiplier problems).

The results are reported in Table 1. The legend of the table is as follows: infeasible method (var $=$ number of variables, eq $=$ number of equalities after slacks are incorporated, bd = number of bounds, it = number of iterations, evals $=$ number of function evaluations); feasible method (var = number of variables, eq $=$ number of equalities, linear $\mathrm{g}=$ number of linear inequality constraints including bounds, nlinear $\mathrm{g}=$ number of nonlinear inequality constraints, it $=$ number of iterations, evals $=$ number of function evaluations). The number of function evaluations counts the associated derivative calls (and accommodates either calls to set the primal-dual data for the systems solves or calls needed to apply Scheme 5.1). When convergence was not 
Table 1 Numerical behavior of infeasible and feasible methods on a set of CUTEr problems

\begin{tabular}{|c|c|c|c|c|c|c|c|c|c|c|}
\hline \multirow[t]{2}{*}{ Problems } & \multicolumn{4}{|c|}{ Infeasible method } & \multicolumn{6}{|c|}{ Feasible method } \\
\hline & $\operatorname{var} \hat{n}$ & eq & bd & it evals & $\operatorname{var} n$ & eq & linear $g$ & nlinear $\mathrm{g}$ & it & evals \\
\hline CHACONN1 & 6 & 3 & 3 & $10 \quad 11$ & 3 & 0 & 0 & 3 & 10 & 25 \\
\hline ERRINBAR & 19 & 9 & 15 & $\alpha<10^{-10}$ & 18 & 8 & 14 & 1 & $\alpha<$ & $10^{-10}$ \\
\hline EXPFITA & 27 & 22 & 22 & $\alpha<10^{-10}$ & 5 & 0 & 0 & 22 & $\alpha<$ & $10^{-10}$ \\
\hline EXPFITB & 107 & 102 & 102 & $\alpha<10^{-10}$ & 5 & 0 & 0 & 102 & $\alpha<$ & $10^{-10}$ \\
\hline HAIFAS & 22 & 9 & 9 & div & 13 & 0 & 0 & 9 & $\operatorname{div}$ & \\
\hline HIMMELP2 & 3 & 1 & 5 & $\alpha<10^{-10}$ & 2 & 0 & 4 & 1 & $\operatorname{div}$ & \\
\hline HIMMELP3 & 4 & 2 & 6 & $\alpha<10^{-10}$ & 2 & 0 & 4 & 2 & 10 & 21 \\
\hline HIMMELP4 & 5 & 3 & 7 & $\alpha<10^{-10}$ & 2 & 0 & 4 & 3 & 10 & 21 \\
\hline HS13 & 3 & 1 & 3 & $i t>200$ & 2 & 0 & 2 & 1 & $\alpha<$ & $10^{-10}$ \\
\hline HS16 & 4 & 2 & 5 & $24 \quad 25$ & 2 & 0 & 3 & 2 & 12 & 26 \\
\hline HS20 & 5 & 3 & 5 & $\alpha<10^{-10}$ & 2 & 0 & 2 & 3 & 12 & 29 \\
\hline HS24 & 5 & 3 & 5 & $\alpha<10^{-10}$ & 2 & 0 & 2 & 3 & $\alpha<$ & $10^{-10}$ \\
\hline HS29 & 4 & 1 & 1 & $12 \quad 13$ & 3 & 0 & 0 & 1 & 15 & 30 \\
\hline HS30 & 4 & 1 & 7 & $\alpha<10^{-10}$ & 3 & 0 & 6 & 1 & 39 & 78 \\
\hline HS32 & 4 & 2 & 7 & $14 \quad 15$ & 3 & 1 & 3 & 1 & $\alpha<$ & $10^{-10}$ \\
\hline HS33 & 5 & 2 & 6 & $i t>200$ & 3 & 0 & 4 & 2 & $\alpha<$ & $10^{-10}$ \\
\hline HS65 & 4 & 1 & 4 & $\alpha<10^{-10}$ & 3 & 0 & 6 & 1 & $\alpha<$ & $10^{-10}$ \\
\hline HS67 & 17 & 14 & 20 & $\alpha<10^{-10}$ & 3 & 0 & 6 & 14 & $i t>$ & 200 \\
\hline HS73 & 6 & 3 & 6 & $\alpha<10^{-10}$ & 4 & 1 & 5 & 1 & $\alpha<$ & $10^{-10}$ \\
\hline HS84 & 8 & 3 & 16 & $\alpha<10^{-10}$ & 5 & 0 & 10 & 6 & $\alpha<$ & $10^{-10}$ \\
\hline HS95 & 10 & 4 & 16 & $\alpha<10^{-10}$ & 6 & 0 & 12 & 4 & $\alpha<$ & $10^{-10}$ \\
\hline HS96 & 10 & 4 & 16 & $\alpha<10^{-10}$ & 6 & 0 & 12 & 4 & $\alpha<$ & $10^{-10}$ \\
\hline HS109 & 13 & 10 & 20 & $\alpha<10^{-10}$ & 9 & 6 & 18 & 2 & $\alpha<$ & $10^{-10}$ \\
\hline HS113 & 18 & 8 & 8 & $i t>200$ & 10 & 0 & 3 & 5 & $\alpha<$ & $10^{-10}$ \\
\hline HS117 & 20 & 5 & 20 & it $>200$ & 15 & 0 & 15 & 5 & $\alpha<$ & $10^{-10}$ \\
\hline HUBFIT & 3 & 1 & 2 & 8 & 2 & 0 & 1 & 1 & 8 & 16 \\
\hline KSIP & 1021 & 1001 & 1001 & 12 & 20 & 0 & 0 & 1001 & 12 & 24 \\
\hline LSQFIT & 3 & 1 & 2 & 9 & 2 & 0 & 1 & 1 & 8 & 16 \\
\hline MATRIX2 & 8 & 2 & 6 & 15 & 6 & 0 & 4 & 2 & $\alpha<$ & $10^{-10}$ \\
\hline MIFFLIN1 & 5 & 2 & 2 & 11 & 3 & 0 & 0 & 2 & 6 & 20 \\
\hline MINMAXBD & 25 & 20 & 20 & $\operatorname{div}$ & 5 & 0 & 0 & 20 & $\alpha<$ & $10^{-10}$ \\
\hline NGONE & 13 & 8 & 14 & $\operatorname{div}$ & 5 & 0 & 8 & 6 & $\alpha<$ & $10^{-10}$ \\
\hline OPTMASS & 77 & 55 & 11 & $\operatorname{div}$ & 66 & 44 & 0 & 11 & 7 & 14 \\
\hline PRODPL0 & 69 & 29 & 69 & $\alpha<10^{-10}$ & 60 & 20 & 60 & 9 & $\alpha<$ & $10^{-10}$ \\
\hline PRODPL1 & 69 & 29 & 69 & $\operatorname{div}$ & 60 & 20 & 65 & 9 & $\alpha<$ & $10^{-10}$ \\
\hline READING4 & 4 & 2 & 8 & $\alpha<10^{-10}$ & 2 & 0 & 4 & 4 & $\alpha<$ & $10^{-10}$ \\
\hline ROSENMMX & 9 & 4 & 4 & $18 \quad 19$ & 5 & 0 & 0 & 4 & 15 & 46 \\
\hline SVANBERG & 20 & 10 & 30 & $\alpha<10^{-10}$ & 10 & 0 & 20 & 10 & 19 & 93 \\
\hline SWOPF & 107 & 102 & 34 & $\alpha<10^{-10}$ & 83 & 78 & 20 & 14 & $\alpha<$ & $10^{-10}$ \\
\hline WOMFLET & 6 & 3 & 3 & $\operatorname{div}$ & 3 & 0 & 0 & 3 & $i t>$ & 200 \\
\hline ZECEVIC2 & 4 & 2 & 6 & $\alpha<10^{-10}$ & 2 & 0 & 4 & 2 & 12 & 29 \\
\hline ZY2 & 5 & 2 & 6 & 78 & 3 & 0 & 4 & 2 & $\alpha<$ & $10^{-10}$ \\
\hline
\end{tabular}


achieved was either because the maximum number of iterations (200) was achieved, or because the step size became too small $\left(\alpha<10^{-10}\right)$, or because the iterates start growing to infinity (div).

Both infeasible and feasible methods converged only for a subset of the problems, given that no globalization strategy was incorporated. In the cases where both converged, the number of iterations was approximately the same. The infeasible method converged for 11 problems whereas the feasible method converged for 15. Actually, this gain in robustness was also observed by looking at the size of the residuals for the problems where both methods did not converge, which showed that the feasible method achieved, in average, smaller residuals.

The procedure to determine the step size for the feasible method (Algorithm 5.1) took a low average number of steps (roughly 2), as we can observe from the difference between the number of function evaluations and the number of iterations taken by this method.

The only problem of the form (1) that satisfies Assumption A4 (concave binding inequalities) is, as far as we could check, problem HS16. The feasible method took much less iterations on this problem than the infeasible method.

\section{Concluding remarks}

Keeping strict feasibility with respect to the inequality constraints in the way required by the feasible primal-dual interior-point method can be numerically affordable. Although the exact computation of the step sizes requires the solution of a number of nonlinear equations per iteration, it is possible to compute them satisfying inexact requirements in a relatively efficient way.

Our numerical findings show that the proposed method can be particularly efficient when the number of inequality constraints is not very large or when the structure of the functions defining the inequality constraints eases the step size calculation considerably. Strict feasibility can be imposed only partially, with a subset of the problem inequalities being treated by slack variables. The inequalities imposed strictly may be those that restrict the objective function domain, or the domain of some other constraint functions.

The most restrictive aspect of the proposed algorithm seems to be the initial point. However, the issue of finding a primal strictly feasible point $\left(g\left(x_{0}\right)<0\right)$ is out of the scope of this paper. See [4], and the references therein, for the determination of feasible points of systems of (nonlinear) equalities and inequalities.

Despite the numerical considerations, looking at the infeasible variant of the primal-dual interior-point method is of interest on itself. It is worth pointing out that the approach presented in this paper covers the infeasible case $([5,14])$ since simple bounds of the type $-x \leq 0$ correspond to concave inequalities. Linear inequality constraints are also concave and can be treated without slack variables for achieving the purpose of fast local convergence. Finally, the observation that the q-quadratic rate is retained in the general nonconcave case provided the angle between the primal step and the gradients of the binding constraints is kept away from ninety degrees, see (28), fits well into the theory of interior-point methods since it corresponds to the notion of centrality. 
Acknowledgements We thank Dominique Orban and two anonymous referees for their comments and suggestions.

\section{References}

1. Anderson, E., Bai, Z., Bischof, C., Blackford, S., Demmel, J., Dongarra, J., DuCroz, J., Greenbaum, A., Hammarling, S., McKenney, A., Sorensen, D.: LAPACK Users' Guide. SIAM, Philadelphia (1999)

2. Byrd, R.H., Liu, G., Nocedal, J.: On the local behaviour of an interior point method for nonlinear programming. In: Numerical Analysis 1997 (Dundee). Pitman Res. Notes Math. Ser., vol. 380, pp. 3756. Longman, Harlow (1998)

3. Coleman, T.F., Li, Y.: On the convergence of interior-reflective Newton methods for nonlinear minimization subject to bounds. Math. Program. 67, 189-224 (1994)

4. Dennis, J.E., El-Alem, M., Williamson, K.: A trust region approach to nonlinear systems of equalities and inequalities. SIAM J. Optim. 9, 291-315 (1999)

5. El-Bakry, A.S., Tapia, R.A., Tsuchiya, T., Zhang, Y.: On the formulation and theory of the Newton interior-point method for nonlinear programming. J. Optim. Theory Appl. 89, 507-541 (1996)

6. Fiacco, A.V., McCormick, G.P.: Nonlinear Programming: Sequential Unconstrained Minimization Techniques. SIAM, Philadelphia (1990)

7. Gould, N.I.M., Orban, D., Sartenaer, A., Toint, P.L.: Superlinear convergence of primal-dual interior point algorithms for nonlinear programming. SIAM J. Optim. 11, 974-1002 (2001)

8. Gould, N.I.M., Orban, D., Toint, P.L.: CUTEr, a constrained and unconstrained testing environment, revisited. ACM Trans. Math. Softw. 29, 373-394 (2003)

9. Heinkenschloss, M., Ulbrich, M., Ulbrich, S.: Superlinear and quadratic convergence of affine-scaling interior-point Newton methods for problems with simple bounds without strict complementarity assumption. Math. Program. 86, 615-635 (1999)

10. Martinez, H.J., Parada, Z., Tapia, R.A.: On the characterization of q-superlinear convergence of quasiNewton interior-point methods for nonlinear programming. Bol. Soc. Mat. Mex. 1, 1-12 (1995)

11. Tits, A.L., Wächter, A., Bakhtiari, S., Urban, T.J., Lawrence, C.T.: A primal-dual interior-point method for nonlinear programming with strong global and local convergence properties. SIAM J. Optim. 14, 173-199 (2003)

12. Vicente, L.N.: Local convergence of the affine-scaling interior-point algorithm for nonlinear programming. Comput. Optim. Appl. 17, 23-35 (2000)

13. Vicente, L.N., Wright, S.J.: Local convergence of a primal-dual method for degenerate nonlinear programming. Comput. Optim. Appl. 22, 311-328 (2002)

14. Yamashita, H., Yabe, H.: Superlinear and quadratic convergence of some primal-dual interior point methods for constrained optimization. Math. Program. 75, 377-397 (1996) 